\title{
High speed pulse generators with electro-optic modulators based on different bit sequence for the digital fiber optic communication links
}

\author{
Mahmoud M. A. Eid ${ }^{1}$, Ashraf S. Seliem², Ahmed Nabih Zaki Rashed ${ }^{3}$, Abd El-Naser A. Mohammed ${ }^{4}$, \\ Mohamed Yassin Ali ${ }^{5}$, and Shaimaa S. Abaza ${ }^{6}$ \\ ${ }^{1}$ Department of Electrical Engineering, College of Engineering, Taif University, Kingdom of Saudi Arabia \\ ${ }^{3,4}$ Electronics and Electrical Communications Engineering Department, Faculty of Electronic Engineering, Menoufia \\ University, Menouf, Egypt \\ ${ }^{2,5,6}$ Benha Faculty of Engineering, Benha University, Benha, Egypt
}

\begin{tabular}{l} 
Article Info \\
\hline Article history: \\
Received Jun 20, 202 \\
Revised Aug 26, 2020 \\
Accepted Sep 10, 202 \\
\hline Keywords: \\
Gaussian \\
Hyperbolic secant \\
Optical modulators \\
Pulse generator (PG) \\
Raised cosine \\
Sine \\
Triangle
\end{tabular}

Article Info

Article history:

Received Jun 20, 2020

Revised Aug 26, 2020

Keywords:

Gaussian

Hyperbolic secant

Pulse generator (PG)

Sine

Triangle

\begin{abstract}
The paper outlines the simulation of various pulse generators for the enhancement of optical fiber access transmission networks within flow rate of $10 \mathrm{Gbps}$ and transmission range of $100 \mathrm{~km}$. The pulse generators are gaussian, hyperbolic secant, triangle, sine, raised cosine in the transmission stage. Proposed pulse generators are mixed with both electro-absorption modulator (EAM) and Mach-Zehnder modulator (MZM) for efficient transmission. We have compared the max. the quality factor with using proposed pulse generators against nonreturn to zero (NRZ) return to zero (RZ) pulse generators in the previous research works for different bit sequences. The signal power amplitude is tested for both optical fiber and PIN photodetector optical time-domain visualizer and RF spectrum analyzer by using in the optimum cases for different bit sequence. It is observed that proposed pulse generators/EAM have presented an efficient increase in Qfactor value compared with proposed pulse generators/MZM for different bit sequences.
\end{abstract}

This is an open access article under the CC BY-SA license.

\section{Corresponding Author:}

Ahmed Nabih Zaki Rashed

Faculty of Electronic Engineering

Menouf, Menoufia University, Egypt

E-mail: ahmed_733@yahoo.com

\section{RELATED WORKS}

They have studied the data error rate optimization using differential phase-shift keying modulation technique [1]. The bit error rate performance is studied for various fiber lengths and the number of optical amplifiers at wavelength of $1550 \mathrm{~nm}$ [2]. The study has outlined the point to point optical communications fiber links [3]. The system consists of multiple quantum well distributed feedback laser and its governing equations with nonreturn to zero code [4-7]. The fiber is modeled with neglecting the Kerr effects. The basic principles and characteristics of photodetection and the bit error rate against the sensibility of the detector [810]. They have presented the performance evaluation of optical fiber communication links [11]. The signal attenuation, bending loss, splices loss, Q-factor value and data error rate are examined for both single-mode and multimode optical fibers at wavelength of $1310 \mathrm{~nm}, 1550 \mathrm{~nm}$ [12]. The complete comparison between different communication channels is studied to ensure the optical fiber channel is the best over other communication channels for high bit rate transmission [13]. The non return to zero (NRZ) and return to zero (RZ) formats are used in performance analysis of long band optical systems at different transmission bit rates 
[14]. The comparison between different formats (NRZ, RZ) is studied at different bit rates. RZ formats show better performance than NRZ at high transmission bit rates. At the wavelength value of $1625 \mathrm{~nm}$ and bit rate value of 35 Gbps, RZ formats give a bit error rate of 5.187 x 10-10 [15].

The bit error rate is enhanced in optical communication systems by using NRZ, RZ pulse generators with different optical modulation techniques [16-20]. NRZ/EAM, NRZ/MZM, RZ/EAM, and RZ/MZM are constructed by using optisystem simulation software in the presence of optical power variations from $15 \mathrm{dBm}$ to $20 \mathrm{dBm}$ in order to achieve the minimum BER values for different 4 bit sequence value of 1010, and 8 bit sequence value of 10101100 [21-25]. The Q-factor and BER values are investigated and stimulated in wavelength division multiplexing networks by using differential phase-shift keying modulation scheme [2630]. The Q-factor and BER are studied against fiber length, gain, number of amplifiers and channel spacing. The trade-off operating parameters is tested to achieve optimized Q-factor and BER [31-36].

This study clarified the max. the quality factor can be enhanced with using triangle pulse generator/EAM for different bit sequences through the comparison with RZ/EAM. As well as the Q-factor is maximized with using hyperbolic Secant pulse generator/MZM through the comparison with NRZ/MZM for 8-bit sequence. Moreover, the Q-factor is maximized with using Gaussian pulse generator/MZM through the comparison with NRZ/MZM for 16-bit sequence.

\section{MODEL DESCRIPTION AND RESEARCH METHOD}

Figure 1 outlines the components of the proposed model: User-defined bit sequence generator generates a bit sequence of 4 bits (1010), 8 bits (10101100), and 16 bits (1100110011001100). The flow rate available up to 10 Gbps through the distance varies from $50 \mathrm{~km}$ to $100 \mathrm{~km}$. Different electrical pulse generators are employed that are namely Gaussian, Triangle, Hyperbolic secant, Sine, and raised cosine pulses with the light signal that is generated from the continuous wave $(\mathrm{CW})$ laser which is both injected to optical modulators.

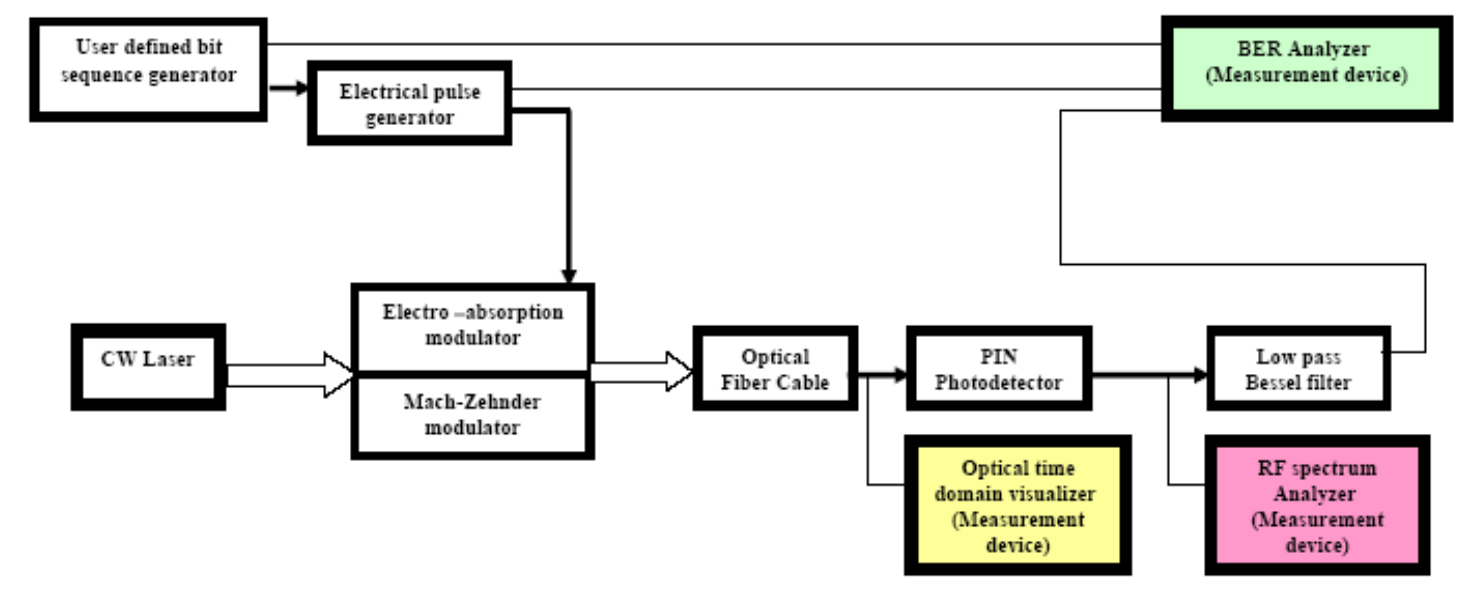

Figure 1. Proposed simulation model

The light signal is forward to fiber cable and then to PIN photodetector where electrical form can be detected. The electrical signal is fed to low pass Bessel filter which is used to remove the ripples from the signal. Both electro-absorption and Mach-Zehnder modulators are used to modulate the electrical and optical signals together. Bit error rate (BER) tests the max Q-factor value and min BER value.

\section{PERFORMANCE ANALYSIS WITH DISCUSSIONS}

We have numerically simulated the different electrical signal pulse generators for high performance of optical access transmission networks. The efficient employment of different electrical pulse generators with optical modulators are done using optisystem simulation program version 13 . The system is built to measure the quality factor based on the efficient coupling between the electrical pulse generator and optical modulators. Based on the clarified parameters in Table 1. The obtained results are assured below:

Figure 2 shows the max. the quality factor with bit period for electrical triangle signal pulse generator with electro-absorption modulator based on 4-bit sequence value of 1010 at a transmission distance of $50 \mathrm{~km}$ and flow rate of $10 \mathrm{Gbps}$. It is found that the max Q-factor reaches to 105.013 and bit error rate 
tends to zero. RF power spectrum after PIN photodetector for triangle pulse generator with electroabsorption modulator based on 4 bits sequence value of 1010 at transmission distance value of $50 \mathrm{~km}$ and flow rate of 10 Gbps is reported in Figure 3. the max signal power amplitude reaches to $-16.9349 \mathrm{dBm}$, and its min value of $103.995 \mathrm{dBm}$. Optical time domain visualizer after optical fiber length of $50 \mathrm{~km}$ for triangle pulse generator with electroabsorption modulator based on 4 bits sequence value of 1010 at transmission distance value of 50 $\mathrm{km}$ and flow rate of $10 \mathrm{Gbps}$ is shown in Figure 4. the max signal power amplitude reaches to $14.114 \mathrm{~mW}$ and its min value reaches to $-0.67209 \mathrm{~mW}$. The bit error rate analyzer for triangle pulse generator with electroabsorption modulator based on eight bits sequence value of 10101100 at transmission distance value of $50 \mathrm{~km}$ and flow rate value of $10 \mathrm{Gbps}$ is shown in Figure 5. Max. Q-factor reaches to 72.4073 and bit error rate tends to zero. RF power spectrum after PIN photodetector for triangle pulse generator with electroabsorption modulator based on 8 bits sequence value of 10101100 at distance value of $50 \mathrm{~km}$ and bit rate value of $10 \mathrm{Gbps}$ is reported in Figure 6. the max signal power amplitude reaches to $-17.2276 \mathrm{dBm}$, while its minimum value reaches to $-103.942 \mathrm{dBm}$. As well as the optical time-domain visualizer after optical fiber length of $50 \mathrm{~km}$ for triangle pulse generator with electro-absorption modulator based on eight bits sequence value of 10101100 at flow rate value of $10 \mathrm{Gbps}$ is reported in Figure 7. The max signal power amplitude reaches to $14.904 \mathrm{~mW}$, while its minimum value reaches to $-0.70792 \mathrm{~mW}$.

Table 1. Variables for the study [13]

\begin{tabular}{|c|c|c|}
\hline Components & Parameter description & Value/Unit \\
\hline $\begin{array}{l}\text { The user-defined bit sequence } \\
\text { generator }\end{array}$ & Bit sequence & $\begin{array}{c}1010,10101100, \\
1100110011001100\end{array}$ \\
\hline Gaussian pulse generator & Amplitude & 1 a.u. \\
\hline Triangle pulse generator & Bias & c0 a.u. \\
\hline Hyperbolic Secant pulse generator & Width & 0.5 bit \\
\hline Cosine pulse generator & Position & 0 bit \\
\hline \multirow[t]{2}{*}{ Sine pulse generator } & Order & 1 \\
\hline & Frequency & $193.1 \mathrm{THz}$ \\
\hline \multirow[t]{3}{*}{ CW Laser } & Power & $20 \mathrm{dBm}$ \\
\hline & Linewidth & $10 \mathrm{MHz}$ \\
\hline & Extinction ratio & $30 \mathrm{~dB}$ \\
\hline \multirow[t]{2}{*}{ Mach-Zehnder modulator } & Symmetry factor & -1 \\
\hline & Modulation index & 0.95 \\
\hline \multirow[t]{3}{*}{ Electroabsorption modulator } & Chirp factor & 0 \\
\hline & Reference wavelength & $1550 \mathrm{~nm}$ \\
\hline & Range & $50 \mathrm{~km}-100 \mathrm{~km}$ \\
\hline \multirow[t]{4}{*}{ ptical fiber } & Attenuation & $0.2 \mathrm{~dB} / \mathrm{km}$ \\
\hline & Dispersion & $16.75 \mathrm{ps} / \mathrm{nm} / \mathrm{km}$ \\
\hline & Differential group delay & $0.2 \mathrm{ps} / \mathrm{km}$ \\
\hline & Responsitivity & $1 \mathrm{~A} / \mathrm{W}$ \\
\hline \multirow[t]{2}{*}{ PIN photodetector } & Dark current & $10 \mathrm{nA}$ \\
\hline & Insertion loss & $0 \mathrm{~dB}$ \\
\hline \multirow{2}{*}{ Low Pass Bessel Filter } & Depth & $100 \mathrm{~dB}$ \\
\hline & Order & 4 \\
\hline
\end{tabular}

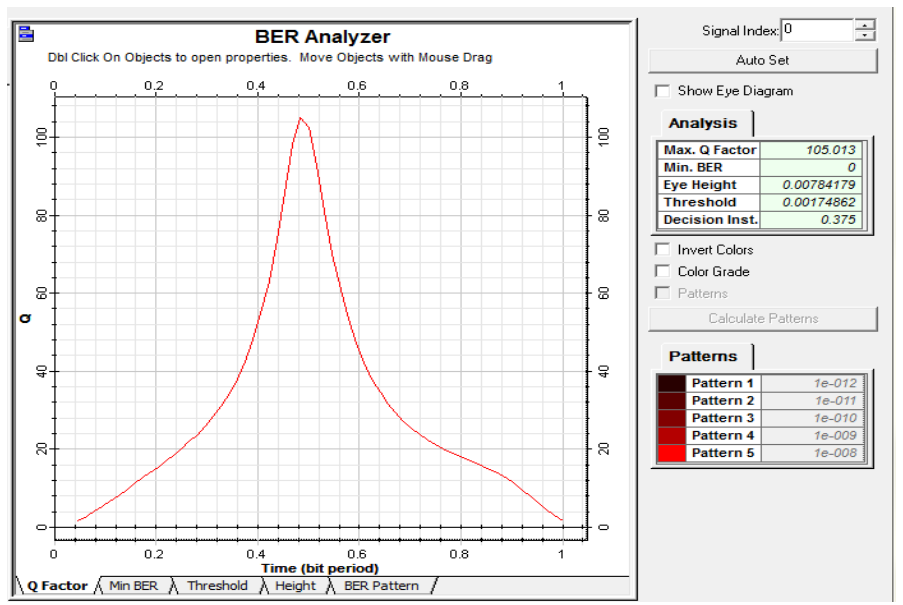

Figure 2. Bit error rate analyzer for triangle pulse generator with electroabsorption modulator based on four bits sequence value of 1010 at transmission distance value of $50 \mathrm{~km}$ 

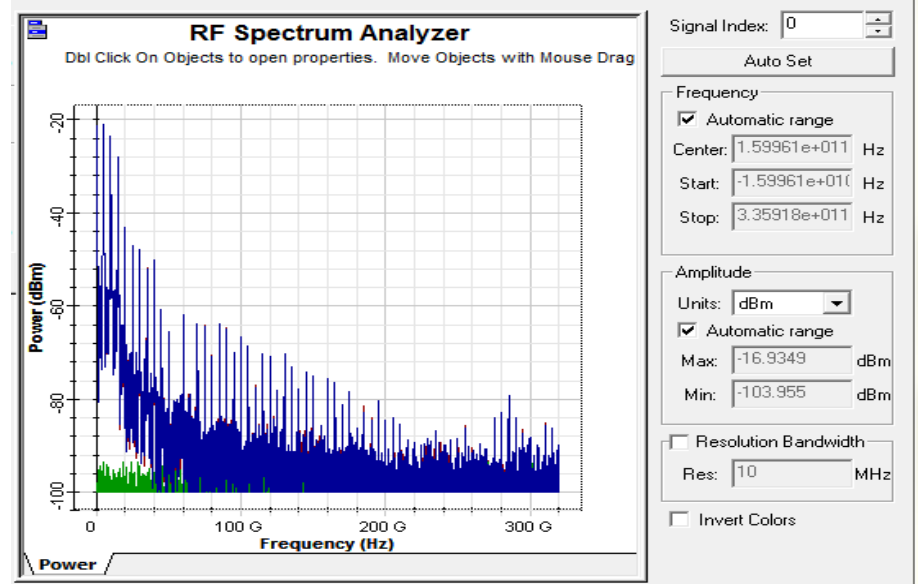

Figure 3. RF power spectrum after PIN photodetector for triangle pulse generator with electroabsorption modulator based on four bits sequence value of 1010 at transmission distance value of $50 \mathrm{~km}$
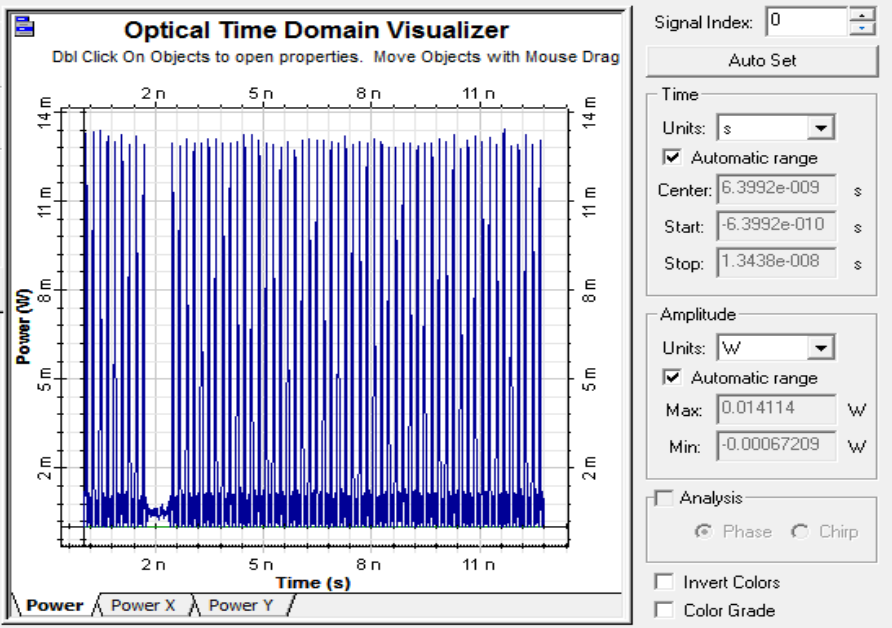

Figure 4. Optical time domain visualizer after optical fiber length of $50 \mathrm{~km}$ for triangle pulse generator with electroabsorption modulator based on four bits sequence value of 1010 at the bit rate value of $10 \mathrm{Gbps}$
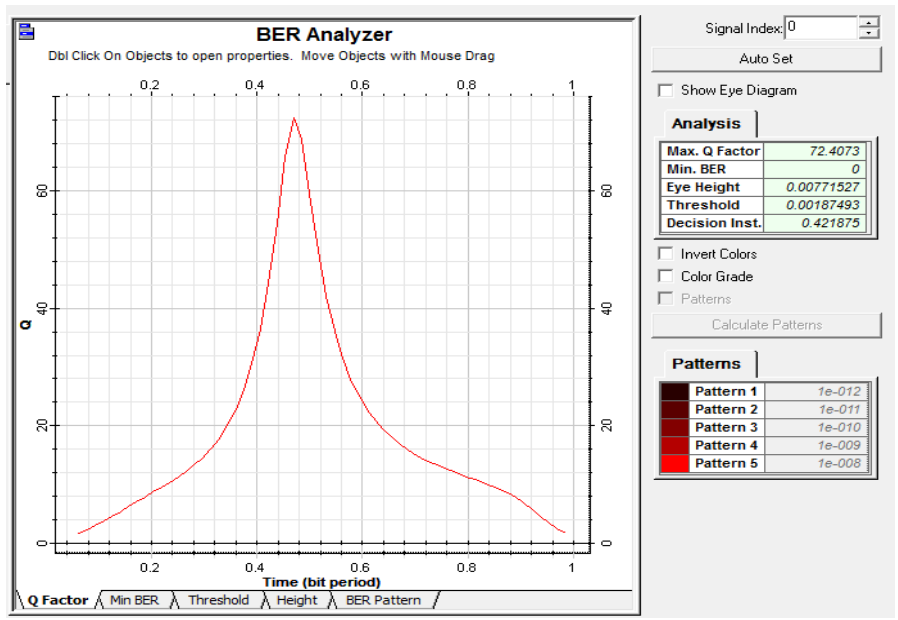

Figure 5. Bit error rate analyzer for triangle pulse generator with electroabsorption modulator based on eight bits sequence value of 10101100 at transmission distance value of $50 \mathrm{~km}$ 

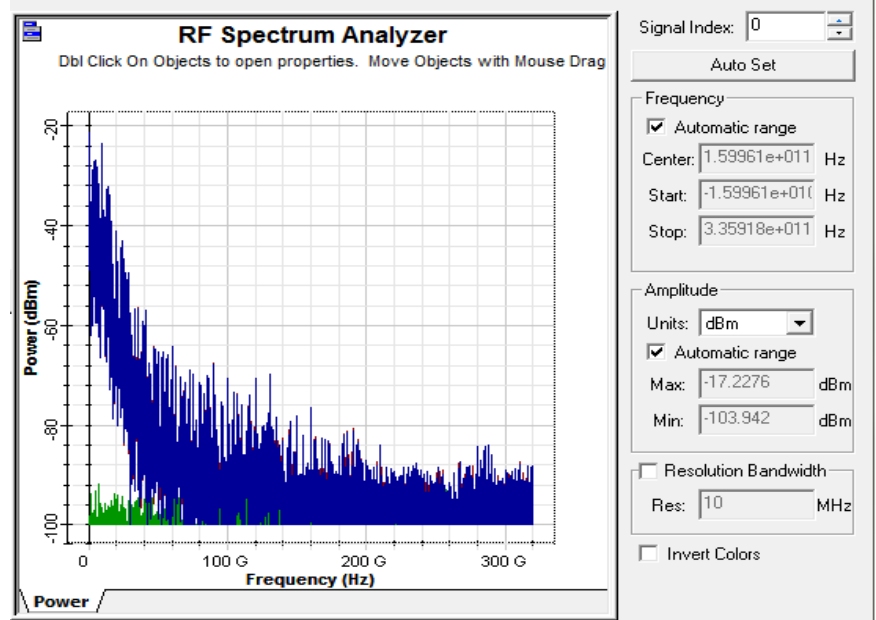

Figure 6. RF power spectrum after PIN photodetector for triangle pulse generator with electroabsorption modulator based on eight bits sequence value of 10101100 at transmission distance value of $50 \mathrm{~km}$
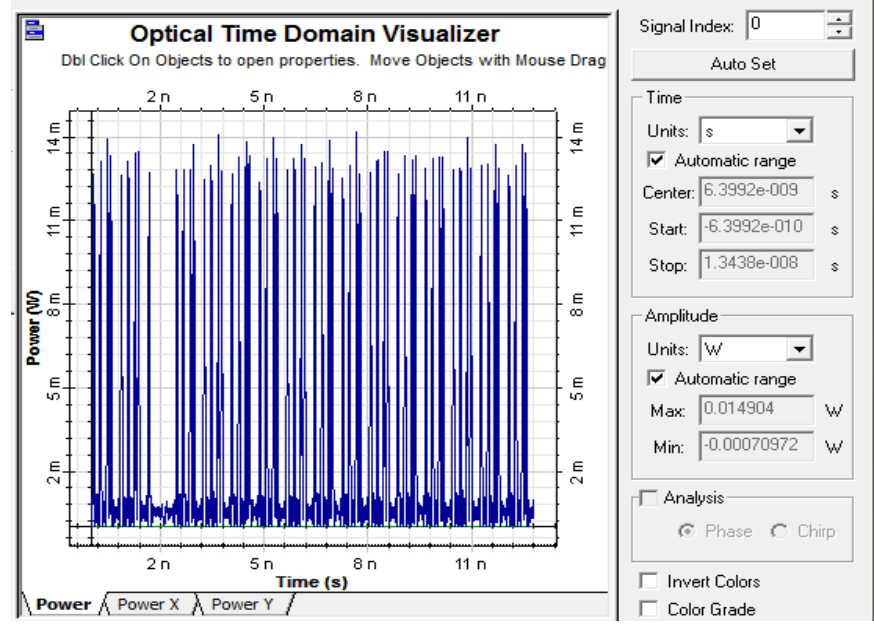

Figure 7. Optical time domain visualizer after optical fiber length of $50 \mathrm{~km}$ for triangle pulse generator with electroabsorption modulator based on eight bits sequence value of 10101100 at flow rate value of $10 \mathrm{Gbps}$

Figure 8 outlines the bit error rate analyzer for triangle pulse generator with electroabsorption modulator based on 16 bits sequence value of 1100110011001100 at transmission distance value of $50 \mathrm{~km}$ and a flow rate of $10 \mathrm{Gbps}$. It is observed that the max Q-factor reaches to 70.5091, and bit error rate tends to zero. RF power spectrum after PIN photodetector for triangle pulse generator with electroabsorption modulator based on 16 bits sequence value of 1100110011001100 at transmission distance value of $50 \mathrm{~km}$ and flow rate of $10 \mathrm{Gbps}$ is outlined in Figure 9. It is indicated that maximum signal power amplitude reaches to $-17.106 \mathrm{dBm}$, and its minimum value reaches to $-103.947 \mathrm{dBm}$.

The time-domain visualizer after optical fiber length of $50 \mathrm{~km}$ for triangle pulse generator with electroabsorption modulator based on sixteen bits sequence value of 1100110011001100 at bit rate value of $10 \mathrm{Gbps}$ is shown in Figure 10. It is evident that the max signal power amplitude reaches to $14.837 \mathrm{~mW}$, and its minimum value is $-0.70654 \mathrm{~mW}$. Figures $11-13$ outline the maximum quality factor against transmission distance variations for different proposed pulse generators/EAM based on different bit sequence values. The negative dramatic effects on the increase of the transmission distance on the maximum signal quality factor. Triangle pulse generator presents the max. Q-factor in compared to either proposed pulse generators or RZ pulse generator [9] for different bit sequence. RF power spectrum after PIN photodetector for Hyperbolic Secant pulse generator with Mach-Zehnder modulator based on 8 bits sequence value of 10101100 at transmission distance value of $50 \mathrm{~km}$ and flow rate of $10 \mathrm{Gbps}$ is shown in Figure 14. It is found that maximum signal power amplitude reaches to $-14.1828 \mathrm{dBm}$ and its min value is $-104.087 \mathrm{dBm}$.

High speed pulse generators with electro-optic modulators based on different bit... (Mahmoud M. A. Eid) 


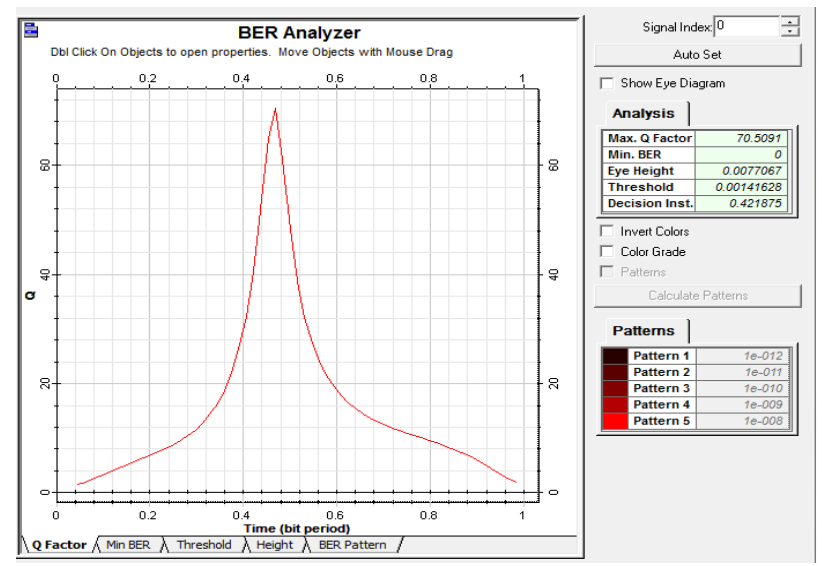

Figure 8. Bit error rate analyzer for triangle pulse generator with electroabsorption modulator based on 16 bits sequence value of 1100110011001100 at transmission distance value of $50 \mathrm{~km}$
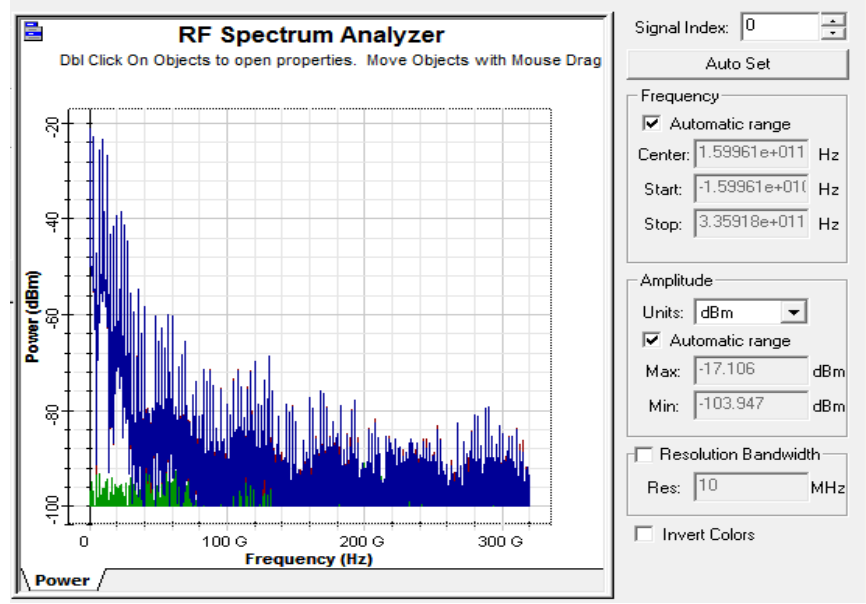

Figure 9. RF power spectrum after PIN photodetector for triangle pulse generator with electroabsorption modulator based on 16 bits sequence value of 1100110011001100 at transmission distance value of $50 \mathrm{~km}$
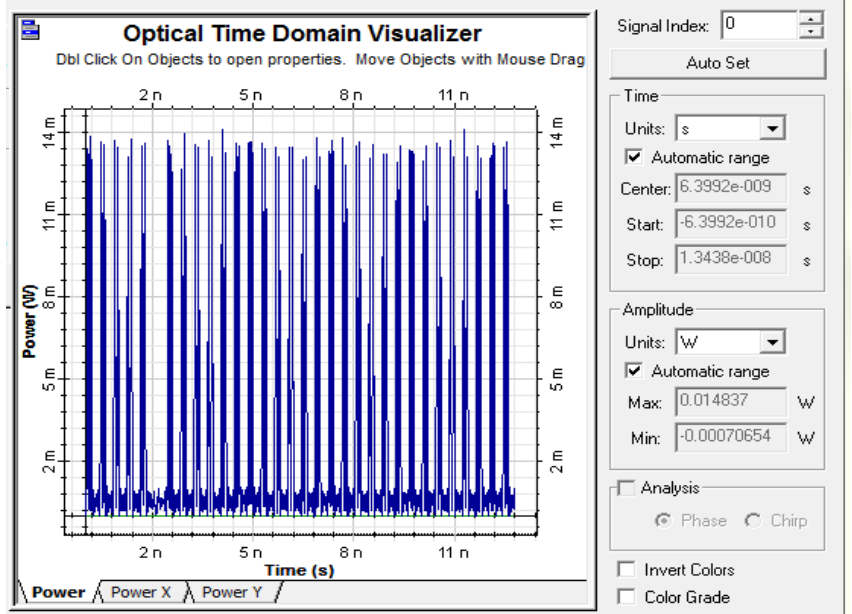

Figure 10. Optical time domain visualizer after optical fiber length of $50 \mathrm{~km}$ for triangle pulse generator with electroabsorption modulator based on 16 bits sequence value of 1100110011001100 at flow rate value of $10 \mathrm{Gbps}$ 


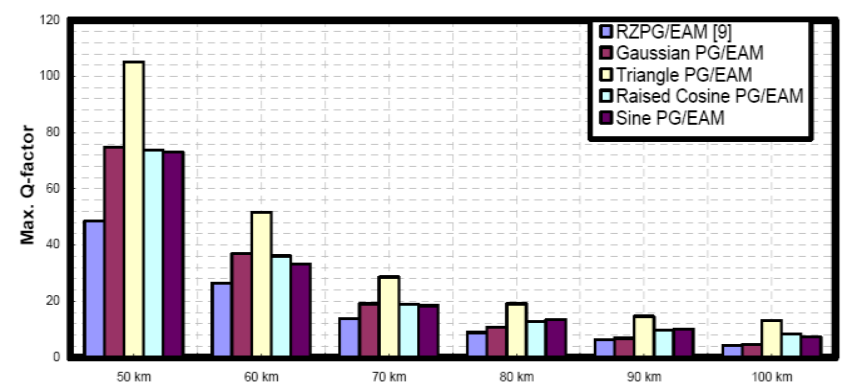

Figure 11. Maximum quality factor versus propagation distance for various proposed pulse generators/EAM based on 4 bits sequence value of 1010

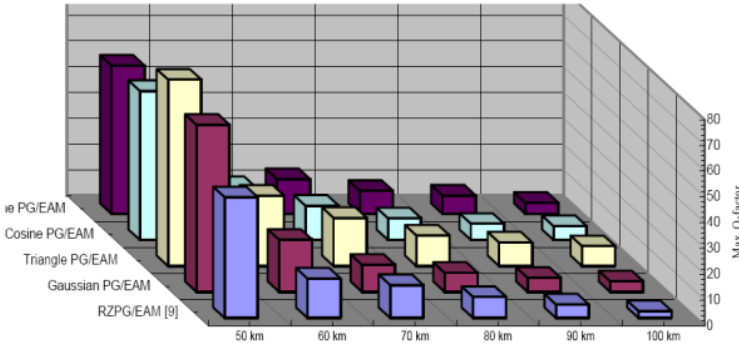

Figure 12. Maximum quality factor with transmission distance for different proposed pulse generators/EAM based on 8 bits sequence value of 10101100

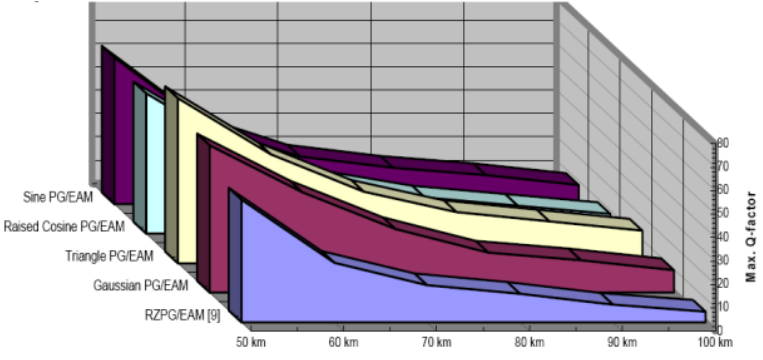

Figure 13. Maximum quality factor with propagation distance for different proposed pulse generators/EAM based on 16 bits sequence value of 1100110011001100
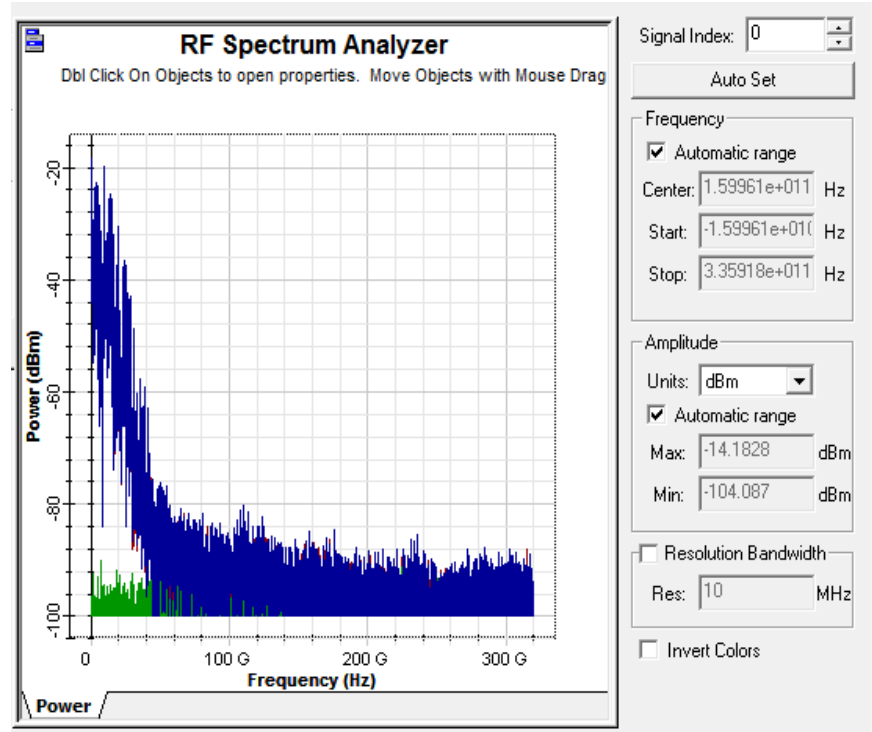

Figure 14. RF power spectrum after PIN photodetector for Hyperbolic Secant pulse generator with MachZehnder modulator based on 8 bits sequence value of 10101100 at transmission distance value of $50 \mathrm{~km}$

The time-domain visualizer after optical fiber length of $50 \mathrm{~km}$ for Hyperbolic Secant pulse generator with Mach-Zehnder modulator based on 8 bits sequence value of 10101100 at flow rate value of 10 Gbps is outlined n Figure 15. Maximum signal power amplitude reaches to $27.892 \mathrm{~mW}$ and its minimum value is $1.3282 \mathrm{~mW}$. As well as the RF power spectrum after PIN photodetector for Gaussian pulse generator with Mach-Zehnder modulator based on sixteen bits sequence value of 1100110011001100 at transmission distance value of $50 \mathrm{~km}$ and flow rate of $10 \mathrm{Gbps}$ is reported in Figure 16. The max signal power amplitude reaches to -

High speed pulse generators with electro-optic modulators based on different bit... (Mahmoud M. A. Eid) 
$14.3078 \mathrm{dBm}$ and its minimum value reaches to $-104.081 \mathrm{dBm}$. Moreover, the time-domain visualizer after optical fiber length of $50 \mathrm{~km}$ for Gaussian pulse generator with Mach-Zehnder modulator based on sixteen bits sequence value of 1100110011001100 at flow rate value of $10 \mathrm{Gbps}$ is reported in Figure 17.

It is found that the max power amplitude reaches to $25.673 \mathrm{dBm}$ and its minimum value reaches to $1.2225 \mathrm{dBm}$. Figures 18 and19 show the variations of max signal quality factor versus distance variations for different proposed pulse generators/MZM based on both 8-bit sequence value of 10101100 and 16-bit sequence value of 1100110011001100 . The dramatic negative effects of the increase of transmission distance on the maximum Q-factor value. It is evident that hyperbolic secant pulse generator/MZM has outlined the maximum signal quality factor in compared with either proposed pulse generators or NRZ PG/MZM [9] for 8-bit sequence value of 10101100. In addition to Gaussian pulse generator/MZM outlines the quality factor with either proposed pulse generators or NRZ PG/MZM [9] for 16-bit sequence value of 1100110011001100.
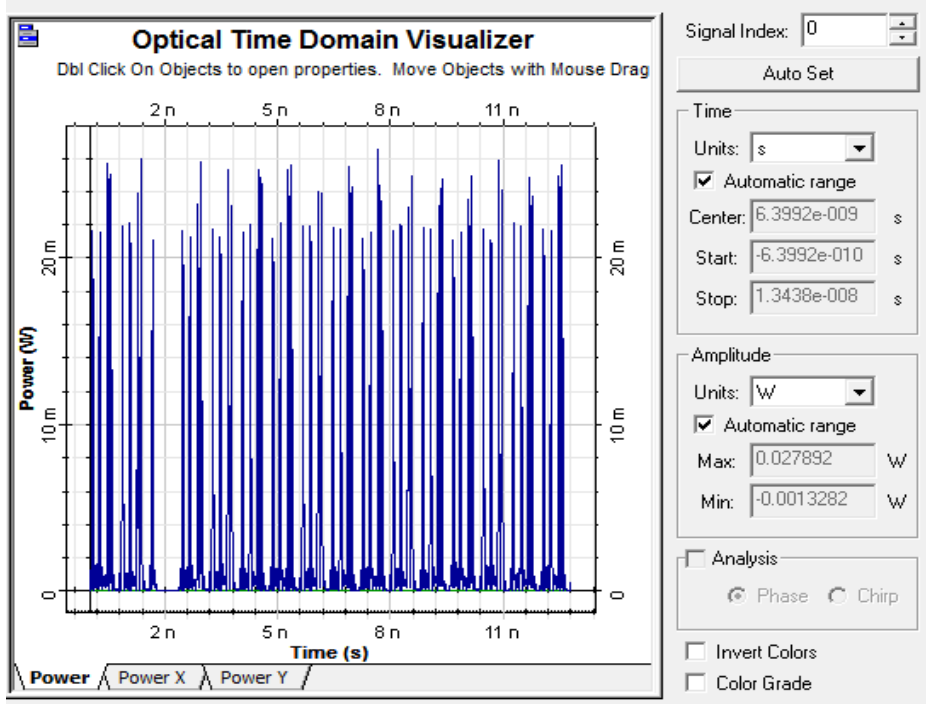

Figure 15. Optical time domain visualizer after optical fiber length of $50 \mathrm{~km}$ for Hyperbolic Secant pulse generator with Mach-Zehnder modulator based on 8 bits sequence value of 10101100 at flow rate value of $10 \mathrm{Gbps}$
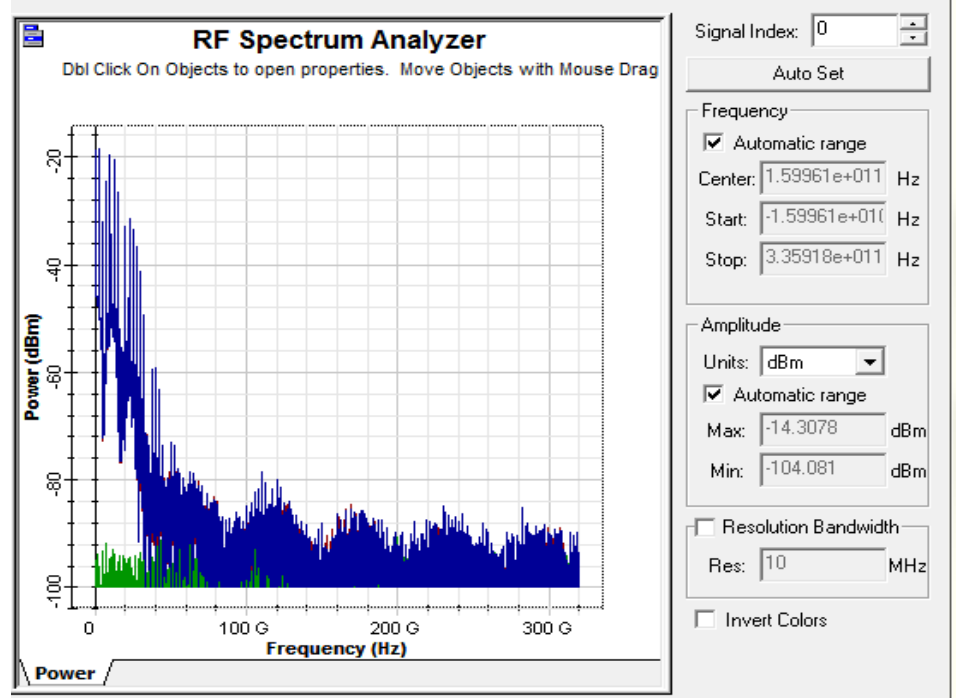

Figure 16. RF power spectrum after PIN photodetector for Gaussian pulse generator with Mach-Zehnder modulator based on 16 bits sequence value of 1100110011001100 at transmission distance value of $50 \mathrm{~km}$ 


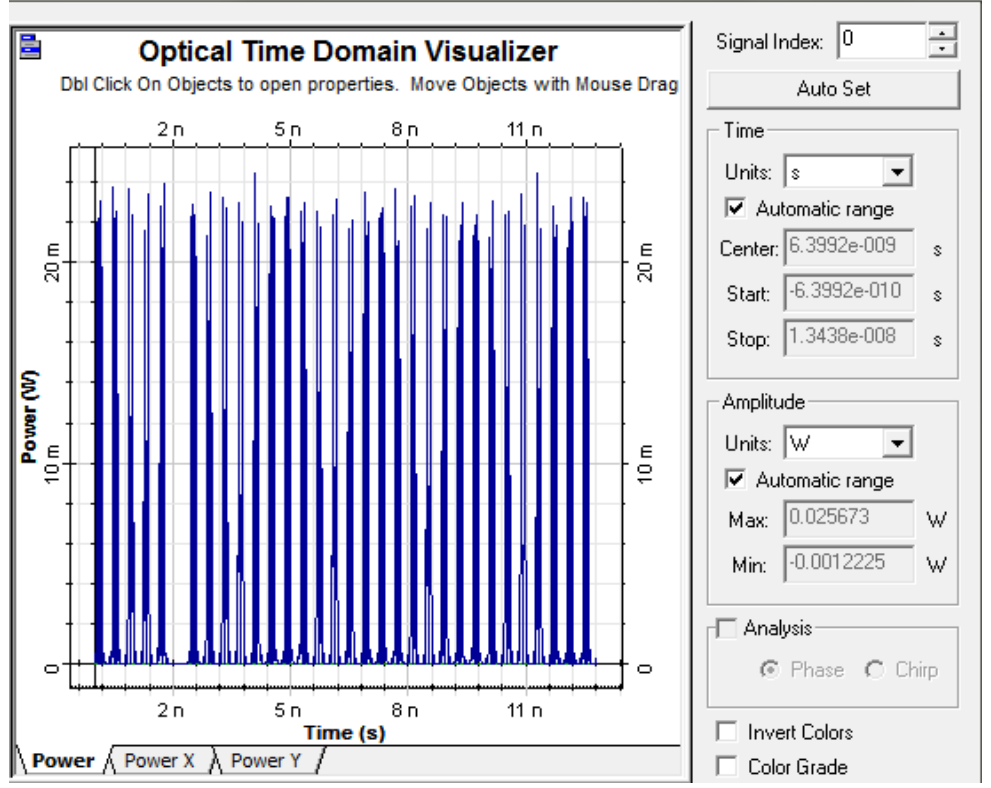

Figure 17. Optical time domain visualizer after optical fiber length of $50 \mathrm{~km}$ for Gaussian pulse generator with Mach-Zehnder modulator based on 16 bits sequence value of 1100110011001100 at flow rate value of $10 \mathrm{Gbps}$

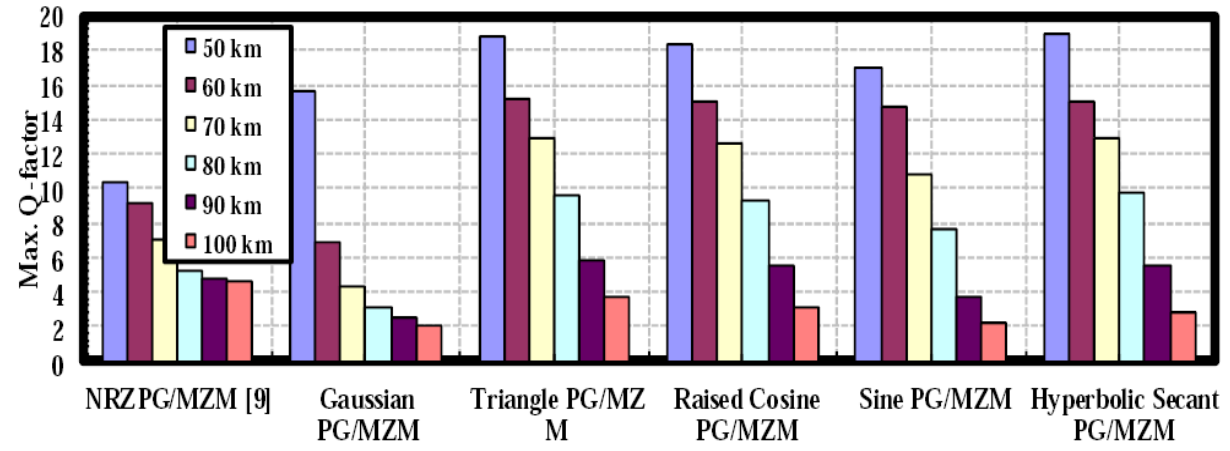

Figure 18. Max signal quality with transmission distance variations for different proposed pulse generators/MZM based on 8 bits sequence value of 10101100

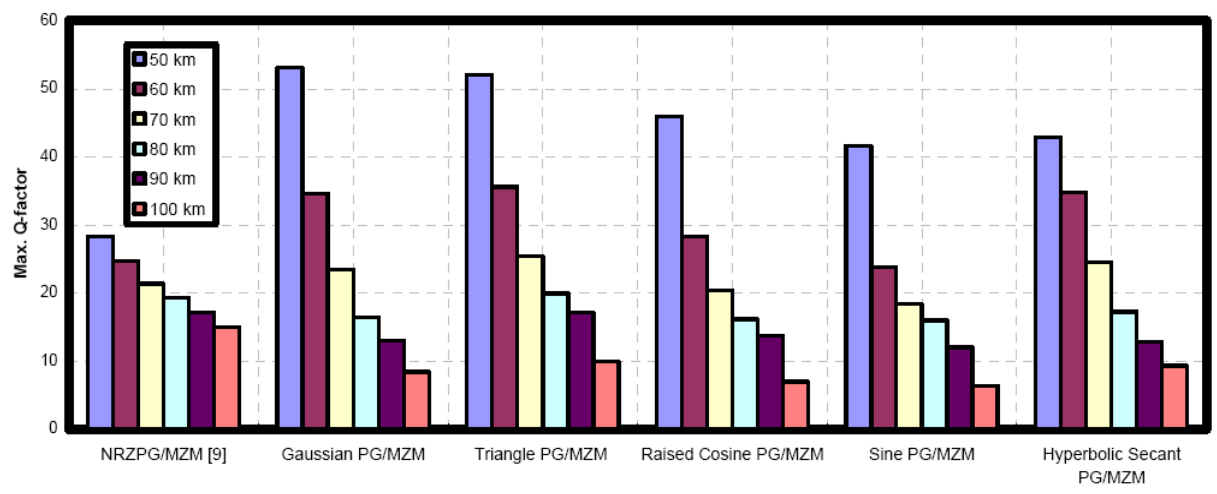

Figure 19. Maximum signal quality factor against transmission distance for different proposed pulse generators/MZM based on 16 bits sequence value of 1100110011001100 


\section{CONCLUSION}

In summary, the efficient coupling between proposed electrical pulse signal generators and optical modulators is suggested in optical access transmission networks. The max quality factor is degraded with the increase of distance based on the numerical simulation. The max. Q-factor value and max. optical signal power is estimated for proposed pulse generators with different optical modulators. Percentage enhancement ratio in max. Q-factor value and max. optical signal power is also estimated in compared with previous pulse generators. It is found that triangle pulse generator/EAM has outlined better both max. Q-factor value, percentage enhancement ratio in max. Q-factor and percentage enhancement ratio in max. optical signal power than either proposed pulse generators/EAM or RZPG/EAM for different bit sequence. As well as hyperbolic-secant PG/MZM has presented better both maxi. Q-factor value, percentage enhancement ratio in max. Q-factor, and percentage enhancement ratio in max. optical signal power than either proposed pulse generators/MZM or NRZPG/MZM for 8-bit sequence. Also, Gaussian PG/MZM has outlined better both max. Q-factor value, percentage enhancement ratio in max. Q-factor and percentage enhancement ratio in max. optical signal power than either proposed pulse generators/MZM or NRZPG/MZM for 16-bit sequence. Therefore it is recommended to use triangle PG/EAM for different bit sequence instead of RZPG/EAM, Hyperbolic-secant PG/MZM instead of NRZPG/MZM for 8-bit sequence, and Gaussian PG/MZM instead of NRZPG/MZM for 16-bit sequence for high-speed transmission networks.

\section{REFERENCES}

[1] M. H. Rubel, S. G. Mahmud, "BER Performance Analysis for Optical Communication using DPSK Modulation," International Journal of Computer Applications, vol. 59, no. 9, pp. 39-44, Dec. 2012.

[2] M. Oukli, et al., "Study and Optimization of High-Bit Rate Optical Fiber Transmission," Serbian Journal Of Electrical Engineering, vol. 5, no. 2, pp. 361-370, Nov. 2008.

[3] S. A. Kadhim, et al., "Performance Evaluation of Experimental Digital Optical Fiber Communication Link," International Journal of Innovative Research in Science, Engineering and Technology, vol. 6, no. 10, pp. 1953419543, Oct. 2017, DOI:10.15680/IJIRSET.2017.0610054.

[4] N. Kaur, G. Kaur, "Complete Performance Analysis of L- band Optical Communication System for NRZ and RZ Format," International Journal of Engineering and Computer Science, vol. 3, no. 5, pp. 5738-5740, May 2014.

[5] K. Kuar, C. Goel, "Threshold based Bit Error Rate Optimization in Four Wave Mixing Optical WDM Systems," American Journal of Engineering Research (AJER), vol. 3, no. 3, pp-225-229, 2014.

[6] W. M. Osman, et al., "Bit Error Rate Performance for Optical Fiber System," Journal of Electrical \& Electronic Systems, vol. 7, no. 1, 2018, DOI: 10.4172/2332-0796.1000250.

[7] S. Jain, and R. Dubey, "A Survey: Optimization of Bit Error Ratio in Fiber Optic Communication System," International Journal of Computer Sciences and Engineering, vol. 3, no. 6, pp. 30-34, 2015.

[8] K. Mukherjee, "Comparative Analysis of Various Optimization Methodologies for WDM System using OptiSystem," International Journal of Innovative Research in Computer and Communication Engineering, vol. 5, no. 10 , Oct. 2017.

[9] S. M. J. Alam, et al., "Improvement of Bit Error Rate in Fiber Optic Communications," International Journal of Future Computer and Communication, vol. 3, no. 4, pp. 281-286, Aug. 2014.

[10] Vandana, G. Pandove, "BER and Q-Value Performance Analysis of WDM Network Using DPSK Modulation Format," International Journal of Advanced Research in Computer Engineering \& Technology (IJARCET), vol. 3, no. 7, pp. 2409-2415, July 2014.

[11] S. Saha, et al., "Optimization Of Gain And Bit Error Rate Of An Erbium Doped Fiber Amplifier For WDM System,” International Journal of Engineering Research \& Technology (IJERT), vol. 2, no. 8, pp. 775-779, Aug. 2013.

[12] S. Alabady, "Simulation and Best Design of an Optical Single Channel in Optical Communication Network," International Arab Journal of e-Technology, vol. 2, no. 2, pp. 91-95, June 2011.

[13] OptiSystem Tutorials, Optical Communication System Design Software version 13, vol. 1, pp. 1-500, 2014, https://dru5cjyjifvrg.cloudfront.net/wp-content/uploads/2015/03/OptiSystem_Tutorials_Volume_1.pdf.

[14] IS Amiri, et al., "Chirped Large Mode Area Photonic Crystal Modal Fibers and its Resonance Modes Based on Finite Element Technique," Journal of Optical Communications, vol. 0, issue 0, Published Online: 23 July 2019, https://doi.org/10.1515/joc-2019-0146.

[15] IS Amiri, et al., "Comparative Simulation of Thermal Noise Effects for Photodetectors on Performance of LongHaul DWDM Optical Networks," Journal of Optical Communications, vol. 0, issue 0, Published Online: 10 August 2019, https://doi.org/10.1515/joc-2019-0152.

[16] IS Amiri, et al., "Single Wide Band Traveling Wave Semiconductor Optical Amplifiers for All Optical Bidirectional Wavelength Conversion," Journal of Optical Communications, vol. 0, no. 0, Published Online: 10 August 2019, https://doi.org/10.1515/joc-2019-0168.

[17] IS Amiri, et al., "Influence of Loading, Regeneration and Recalling Elements Processes on the System Behavior of All Optical Data Bus Line System Random Access Memory," Journal of Optical Communications, vol. 0, no. 0, Published Online: 15 August 2019, https://doi.org/10.1515/joc-2019-0163. 
[18] Ahmed Nabih Zaki Rashed, et al., "The effect of using different materials on erbium-doped fiber amplifiers for indoor applications," Results in Physics, vol. 15, paper id: 102650, Dec. 2019, https://doi.org/10.1016/j.rinp.2019.102650.

[19] Z. Ramezani, et al., "A novel symmetric GaN MESFET by dual extra layers of Si3N4," Physica E: LowDimensional Systems and Nanostructures Journal, vol. 70, pp. 135-140, 2015.

[20] A Orouji, et al., "High-performance SOI MESFET with modified depletion region using a triple recessed gate for RF applications," Materials Science in Semiconductor Processing Journal, vol. 30, pp. 545-553, 2015.

[21] Z. Ramezani, A. A Orouji, "Investigation of veritcal graded channel doping in nanoscale fully-depleted SOIMOSFET", Superlattices and Microstructures Journal, vol. 98, pp. 359-370, 2016.

[22] M. K Anvarifard, et al., "Proposal of an Embedded Nanogap Biosensor by a Graphene Nanoribbon Field Effect Transistor for Biological Samples Detection," Physica Status Solidi (a), vol. 0, no. 0, Article 1900879, Dec. 2019.

[23] M. K Anvarifard, et al., "A Nanoscale Modified band energy junctionless transistor with considerable progress on the electrical and frequency issue" Materials Science in Semiconductor Processing Journal, vol. 107, Article 104849, 2020.

[24] Z. Ramezani, et al.,, "A Nano junctionless Double-Gate MOSFET by Using the Charge Plasma Concept to Improve Short-Channel Effects and Frequency Characteristics", Journal of Electronic Materials, vol. 48, no. 11, pp. 74877494, 2019.

[25] IS Amiri and Ahmed Nabih Zaki Rashed, "Power Enhancement of the U-shape Cavity Microring Resonator Through Gap and Material Characterizations," Journal of Optical Communications, vol. 0, no. 0, Published Online: 19 Oct. 2019, https://doi.org/10.1515/joc-2019-0108.

[26] IS Amiri, et al., "The Engagement of Hybrid Ultra High Space Division Multiplexing with Maximum Time Division Multiplexing Techniques for High-Speed Single-Mode Fiber Cable Systems," Journal of Optical Communications, vol. 0, no. 0, Published Online: 19 Oct. 2019, https://doi.org/10.1515/joc-2019-0205

[27] Ahmed Nabih Zaki Rashed, et al., "Polar Polarization Mode and Average Radical Flux Intensity Measurements Based on All Optical Spatial Communication Systems," Journal of Optical Communications, vol. 0, no. 0, Published Online: 19 Oct. 2019, https://doi.org/10.1515/joc-2019-0159.

[28] Ahmed Nabih Zaki Rashed, et al., "High-Speed Transmission Circuits Signaling in Optical Communication Systems," Journal of Optical Communications, vol. 0, no. 0, Published Online: 9 Nov. 2019, https://doi.org/10.1515/joc-2019-0197.

[29] IS Amiri, et al., "Technical Specifications of the Submarine Fiber Optic Channel Bandwidth/Capacity in Optical Fiber Transmission Systems," Journal of Optical Communications, vol. 0, no. 0, Published Online: 9 Nov. 2019, https://doi.org/10.1515/joc-2019-0226.

[30] IS Amiri, Ahmed Nabih Zaki Rashed, "Signal Processing Criteria Based on Electro-Optic Filters for Fiber Optic Access Transceiver Systems," Journal of Optical Communications, vol. 0, no. 0, Published Online: 12 Nov. 2019, https://doi.org/10.1515/joc-2019-0116.

[31] Ahmed Nabih Zaki Rashed, et al., "Pump Laser Automatic Signal Control for Erbium-Doped Fiber Amplifier Gain, Noise Figure, and Output Spectral Power," Journal of Optical Communications, vol. 0, no. 0, Published Online: 17 Dec. 2019, https://doi.org/10.1515/joc-2019-0203.

[32] IS Amiri, et al., "Performance Enhancement of Fiber Optic and Optical Wireless Communication Channels by Using Forward Error Correction Codes," Journal of Optical Communications, vol. 0, no. 0, Published Online: 24 Dec. 2019, https://doi.org/10.1515/joc-2019-0191.

[33] IS Amiri, et al., "Optical Communication Transmission Systems Improvement Based on Chromatic and Polarization Mode Dispersion Compensation Simulation Management", Published online 23 November 2019, Optik Journal, vol. 207, article163853, April 2020, https://doi.org/10.1016/j.ijleo.2019.163853.

[34] IS Amiri, et al., "Analytical Model Analysis of Reflection/Transmission Characteristics of Long-Period Fiber Bragg Grating (LPFBG) by Using Coupled Mode Theory," Journal of Optical Communications, vol. 0, no. 0, Published Online: 2 April 2020, https://doi.org/10.1515/joc-2019-0187.

[35] IS Amiri, Ahmed et al., "Conventional/Phase Shift Dual Drive Mach-Zehnder Modulation Measured Type Based Radio over Fiber Systems,” Journal of Optical Communications, vol. 0, no. 0, Published Online: 14 April 2020, https://doi.org/10.1515/joc-2019-0312.

[36] Ahmed Nabih Zaki Rashed, et al., "RZ Line Coding Scheme With Direct Laser Modulation for Upgrading Optical Transmission Systems," Open Eng. Journal, vol. 10, no. 1, pp. 546-551, 2020, https://doi.org/10.1515/eng-20200066 . 